\title{
ULTRASONOGRAPHY OF HANDS AND WRISTS IN PATIENTS WITH RHEUMATOID ARTHRITIS WITH SUSPECTED DISEASE ACTIVITY
}

Rafaela Regina Silva ${ }^{1}$, Raquel Garcia Braga ${ }^{1}$, Bruno Bordallo Corrêa ${ }^{1}$, Camila da Silva Ventura ${ }^{1}$, Eduardo Diniz de Castro Menezes ${ }^{1}$, Luiza Catharina Brusasco Grandini ${ }^{1}$, Marcus Vinicius de Jesus da Silva ${ }^{1}$, Ingrid Bandeira Moss ${ }^{1, \star}$, Blanca Elena Rios Gomes Bica ${ }^{1}$

1.Universidade Federal do Rio de Janeiro, Rio de Janeiro (RJ), Brazil.

*Corresponding author: guidmoss.im@gmail.com

\section{BACKGROUND}

Rheumatoid arthritis (RA) is an autoimmune disease that affects the synovial membrane of small joints, which can cause cartilage damage and bone erosion. Ultrasonography with power Doppler (PD) is an important complementary diagnostic and follow-up method for these patients, especially for those in whom the clinical assessment is doubtful and disease activity indices may not clearly and objectively reflect the status of the disease. The objective of this study was to describe joint involvement due to active disease in patients with inconclusive clinical examination in routine visit.

\section{METHODS}

Hands and wrist ultrasonography were evaluated in patients diagnosed with established RA whose physical examination raised doubts about the real presence of active disease during routine visit in order to assess the presence and the degree of synovitis by PD, as well as the most affected joints.

\section{RESULTS}

Exams from 20 patients were selected, with a total of 112 joints evaluated. Ultrasonographic signs of inflammation were detected in 11 patients (55\%), resulting in 90 joints exhibiting some degree of synovitis. Seven patients (35\%) had grade 1 PD synovitis (mild) in at least one joint, while $6(30 \%)$ had grade 2 PD (moderate) and 1 patient had grade 3 (severe). The most affected joint was the second metacarpophalangeal joint, followed by the wrist and third metacarpophalangeal.

\section{CONCLUSION}

Although clinical criteria for assessing disease activity have evolved over time, in real-life clinical practice we often have difficulty in assessing the presence of active synovitis on physical examination, especially in patients with long-term disease and multiple deformities and in those whose metrics can be influenced by other associated conditions, such as fibromyalgia and osteoarthritis. Numerous studies describe the utility of ultrasonography in this context, complementing the clinical assessment, since the relationship between subclinical synovitis, presence of positive PD and evolution with structural joint damage is well established. In patients with clinically evident synovitis, the presence of ultrasound activity is predictable, which may not occur in patients whose physical examination is doubtful. The results found demonstrate that there is evidence of active inflammation in about half of the patients included, and in one third a moderate degree of synovitis was found. We believe that the data obtained reinforce the importance of ultrasonography in monitoring the inflammatory activity and therapeutic efficacy, with the aim of avoiding evolution of structural damage and minimizing the need to increase immunosuppression in patients with inactive disease.

\section{KEYWORDS}

Ultrasonography, Rheumatoid arthritis, Monitoring, Ultrasound, Power Doppler. 